\title{
Processus d'intégration des migrants Mbororo à l'économie urbaine : cas de Bafoussam et Dschang (Ouest-Cameroun)
}

\author{
Linda Pamela Kamgne Kamwa, (Doctorante, Géographie) \\ Maurice Tsalefac, (Professeur des Universités, Climatologue) \\ Département de Géographie, Faculté des Lettres et Sciences Humaines, \\ Université de Dschang, Dschang, Cameroun
}

Doi:10.19044/esj.2020.v16n17p155 URL:http://dx.doi.org/10.19044/esj.2020.v16n17p155

\section{Résumé}

Présents sur la quasi-totalité du territoire camerounais, les Mbororo sont un peuple d'éleveurs semi-nomades qui résident principalement en zone rurale. Cependant, leur espace vital traditionnel étant de plus en plus saturé (multiplication des hommes et par là réduction de l'espace pastoral) et en proie à des conflits, ils sont nombreux qui migrent pour la ville où ils se sédentarisent. L'objectif de cette étude est de présenter le processus d'intégration des migrants Mbororo à l'économie urbaine à Bafoussam et à Dschang. Des enquêtes ont été menées auprès de 270 Mbororo dans la ville de Bafoussam et 230 dans la ville de Dschang. Elles ont été conduites à l'aide de questionnaires individuels. Les résultats montrent que nombreux sont les Mbororo qui se lancent dans le secteur informel, principalement dans le commerce, ceci à cause de la sous-scolarisation, du frein de la langue, de l'absence de qualification professionnelle et de financement. Il se met en place afin de faciliter cette intégration des processus de sédentarisation qui passent par l'auto-emploi, l'entraide familiale et les candidatures spontanées. Pour simplifier le processus de sédentarisation dans les activités urbaines de ce peuple rural, il s'avère impératif davantage vulgariser les formations académique et professionnelle (en campagne et en ville) et enfin, faciliter et simplifier les emprunts bancaires par toutes les couches sociales, pour permettre plus d'auto-emplois et par là la création d'emplois.

Mots-clés : Bafoussam, Dschang, Mbororo, sédentarisation, ville 


\title{
Integration process of Mbororo migrants in the urban economy: the case of Bafoussam and Dschang (West-Cameroon)
}

\author{
Linda Pamela Kamgne Kamwa, (Doctorante, Géographie) \\ Maurice Tsalefac, (Professeur des Universités, Climatologue) \\ Département de Géographie, Faculté des Lettres et Sciences Humaines, \\ Université de Dschang, Dschang, Cameroun
}

\begin{abstract}
Present on almost all the Cameroonian territory, Mbororos are a people of semi-nomadic breeders who live mainly in rural areas. However, their traditional living space being more and more saturated (multiplication of men and thereby reduction of pastoral space) and in the grip of conflicts, many of them migrate to the city where they settle down. The objective of this study is to present the integrating process of Mbororo migrants into the urban economy in Bafoussam and Dschang. Surveys were conducted with 270 Mbororos in the city of Bafoussam and 230 in the city of Dschang. They were conducted using individual questionnaires. The results show that many Mbororos enter into the informal sector, mainly into trade, because of under-education, language barrier, lack of professional qualification and funding. Sedentarisation processes which include self-employment, family support and spontaneous applications are being put in place to facilitate this integration. To simplify the sedentarisation process in the urban activities of this rural people, it is imperative to put more emphasis on the importance of schooling, to encourage various professional training and finally to grant and simplify access to funding, to allow more self-employment and thereby job creation.
\end{abstract}

Keywords: Bafoussam, Dschang, Mbororos, sedentarisation, city

\section{Introduction}

Les Mbororo sont un peuple rural appartenant au groupe Peul/Fulani. L'élément phare de caractérisation de ce peuple est le bétail c'est-à-dire les bœufs. De fait, les vaches constituent un critère de différenciation entre les différents clans. L'image attachée au Peul est celui d'un pasteur, incapable de s'adonner aux travaux des champs. En effet, « les Mbororo affirment souvent qu'ils ne connaissent que le travail auprès des animaux et ils s'avouent incapables de toute autre activité »(Boutrais, 1996). Toutefois, avec la 
mondialisation et la crise du monde rural, le taux d'exode rural augmente considérablement en Afrique et la population Mbororo camerounaise ne fait pas exception à ce phénomène. En raison des opportunités d'emploi et de scolarisation, les villes exercent une forte attraction sur ce peuple seminomade (Boutrais, 1994).

Le problème qui se pose dès lors est celui de la sédentarisation des peuls Mbororo dans les activités urbaines. Plus spécifiquement comment ce peuple rural et semi-nomade s'intègre t'il à l'économie urbaine ? En effet, sur la corne de l'Afrique, les Mbororo se lancent dans le commerce moderne et dans les circuits d'importation et de distributions informels (Piguet, 1998). Ils sont des gardiens de maisons, vendeurs de bijoux, ou fermiers dans certains cas, avec des activités dans les transports et le petit commerce (vente du thé, café moulu, turbans, objets d'art) (ME de Bruijn et al., 2011). En Mongolie, ces derniers travaillent sept mois par an sur les sites miniers du désert de Gobi (Gente, 2013). Qu'en est-il dans les villes étudiées : Bafoussam et Dschang? Quelles sont les activités exercées par les Mbororo dans ces deux villes ? En outre, l'accès et la pratique de ces métiers semblent commodes aux Mbororo. On s'interroge dès lors sur les stratégies d'intégration mises en place par ces derniers. De plus, pour un peuple rural, incapable de toute autre activité que l'élevage et qui se sédentarise en milieu urbain, ne rencontrent t-ils pas des obstacles dans cette marche d'intégration ? Si oui lesquels ?

Ce travail s'effectue dans les villes de Bafoussam et Dschang à l'Ouest Cameroun, où 270 Mbororo ont été enquêté à Bafoussam et 230 à Dschang, ceci à l'aide des questionnaires et de focus group discussion. L'objectif de cette étude est de présenter le processus d'intégration des migrants Mbororo à l'économie urbaine. De manière spécifique, il est question de mettre en évidence les métiers exercés par les Mbororo migrants en ville, les stratégies d'intégration mises en place par ces derniers et enfin les obstacles qu'ils rencontrent dans ce processus de sédentarisation aux activités urbaines.

\section{Méthodologie}

L'étude s'est effectuée dans les villes de Bafoussam et Dschang dans la région de 1'Ouest-Cameroun. La ville de Bafoussam est située sur les Hautes-Terres de $1^{\prime}$ Ouest, entre les latitudes $5^{\circ} 26^{\prime}$ et $5^{\circ} 31^{\prime}$ Nord; les longitudes $10^{\circ} 20^{\prime}$ et $10^{\circ} 30^{\prime}$ Est, pour une superficie de 2600 hectares et une population de 360745 habitants en 2005 (Mpakam et Al., 2006). Elle est subdivisée en trois arrondissements : Bafoussam $\mathrm{I}^{\mathrm{er}}$, Bafoussam II ${ }^{\mathrm{ème}}$ et Bafoussam III ème (Figure 1). L'industrie est embryonnaire, par conséquent elle est très peu génératrice d'emplois. Le secteur privé dominé par la composante informelle est le principal pourvoyeur d'emplois. Seulement 30\% de la population active exercent des métiers formels (Mpakam et Al., 2006). La ville de Dschang quant à elle s'étend sur une superficie de $262 \mathrm{~km}^{2}$, entre les 
parallèles $5^{\circ} 25^{\prime}$ et $5^{\circ} 30^{\prime}$ de latitude Nord et les méridiens $10^{\circ}$ et $10^{\circ} 05^{\prime}$ de longitude Est. Le dernier recensement 2005 estimait sa population à 70000 habitants. Elle pourrait atteindre les 100000 habitants en 2010, si le taux de croissance de 4,7\% est maintenu. L'espace urbain couvre une superficie de 5655 ha à l'intercession des territoires des chefferies Foto et Foréké-Dschang (Figure 2). C'est une ville universitaire dont l'économie est basée sur l'agriculture, l'élevage, l'artisanat et le commerce (ERA, 2006).

Le choix s'est porté sur ces deux villes pour un double raison: Premièrement, la migration des pasteurs Mbororo est un phénomène rependu sur l'ensemble du territoire camerounais, car le monde rural traverse une crise générale. L'option s'est donc orientée sur les villes proches de la zone d'attache de la plupart d'entre eux, qui est la région du Nord-Ouest. Ensuite, il est question d'établir une comparaison entre la ville de Bafoussam et celle de Dschang, afin de déterminer si le processus d'intégration à l'économie urbaine des Mbororo varie d'une ville à l'autre ou si elle est similaire dans toutes les villes.

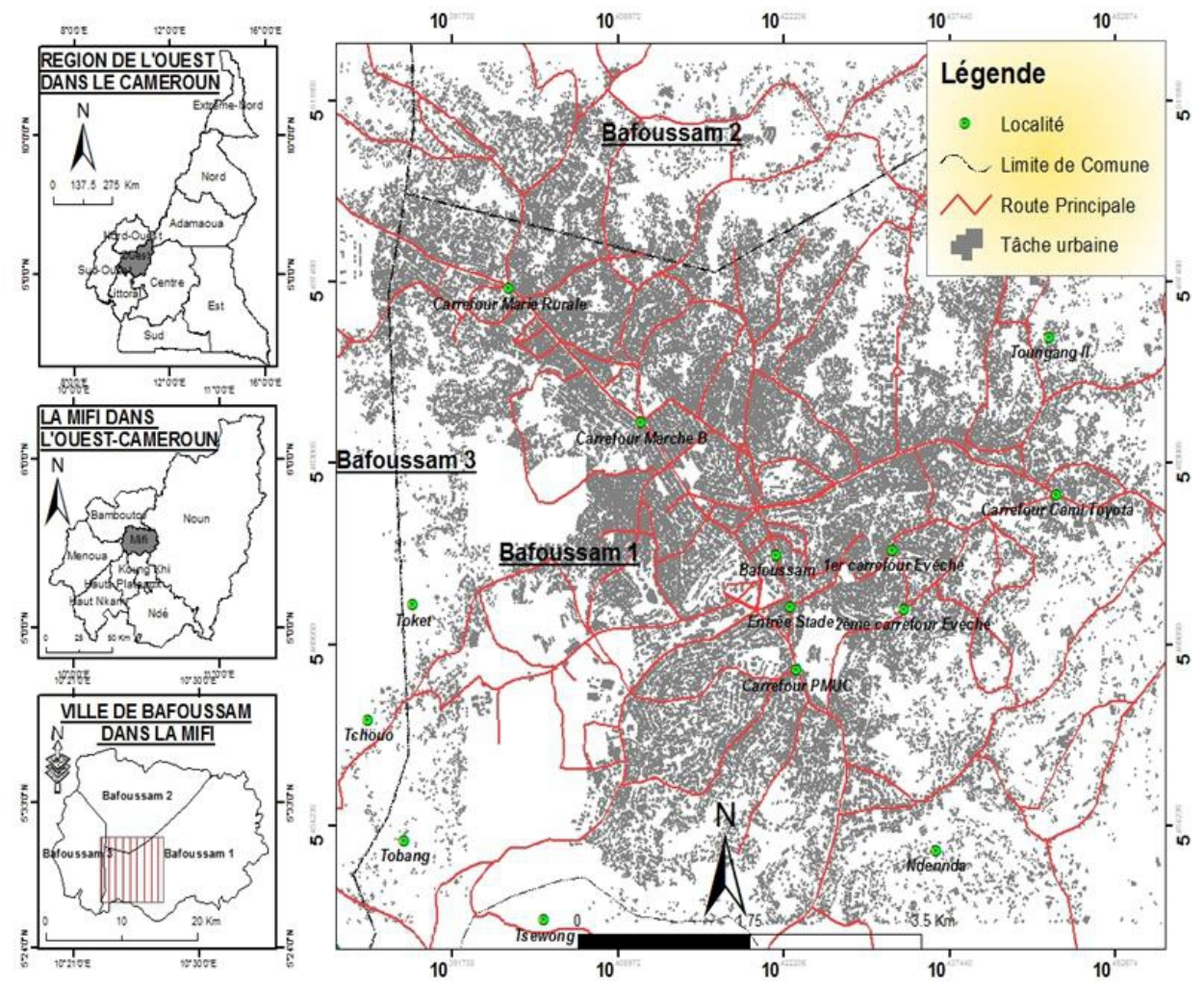

Figure 1 : Carte de la ville de Bafoussam avec les arrondissements d'étude 


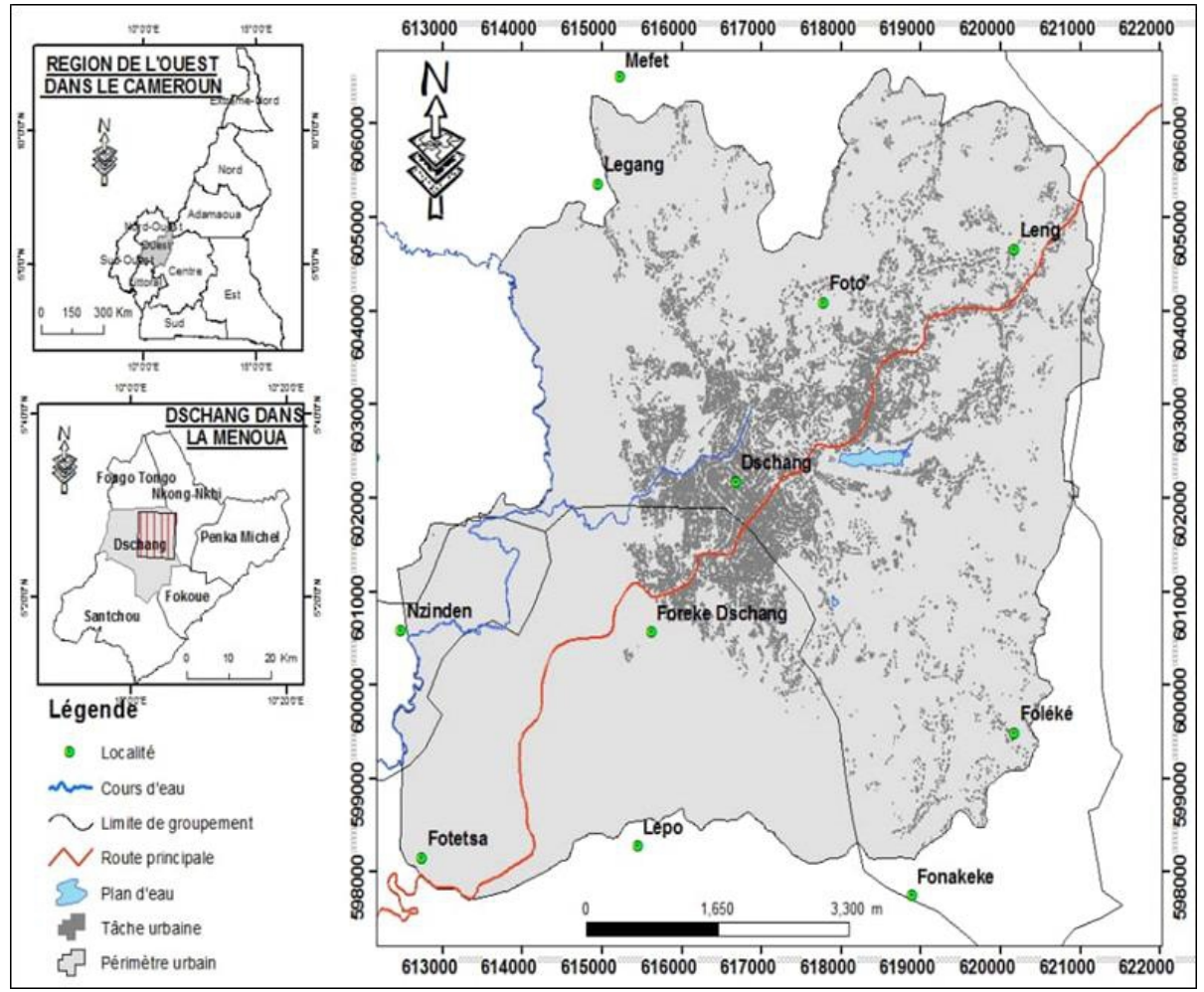

Figure 2 : Carte du périmètre urbain de Dschang

L'ensemble du travail est basé sur l'utilisation de la méthode hypothético-déductive, fondée sur l'émission des hypothèses qui ont été par la suite vérifiées à travers des observations sur le terrain puis, confirmées ou infirmées. Au préalable, l'observation documentaire nous a permis dans le cadre de cette étude de mieux circonscrire le travail en s'appuyant sur des travaux antérieurs. Par la suite, il a fallu réaliser un travail de terrain, qui a consisté à aller auprès de la population cible (les hommes, femmes, jeunes et vieillards Mbororo).

Au préalable, il a fallu passer par des informateurs clés tels que les chefs de quartier et le représentant Mbororo (l'Ardo), qui sont des personnes propices ayant des données sur la présence et le nombre éventuel de Mbororo par quartiers. Les informations ont été recueillies aux moyens d'outils comme le guide d'entretien, le questionnaire et le focus group discussion. En l'absence de données statistiques exactes sur le nombre de Mbororo au Cameroun et plus particulièrement à Bafoussam et à Dschang, nous avons eu recours à l'échantillonnage par quotas. Nous avons aussi employé l'échantillonnage par boule de neige, dans la mesure où ce sont les Mbororo enquêtés qui nous mettaient en contact avec d'autres Mbororo, en nous indiquant leur lieu d'habitat. Les Mbororo se repartissent sur l'ensemble des deux territoires enquêtés (Bafoussam et Dschang), avec une majorité toutefois installée dans 
les quartiers abritant des mosquées. Les questionnaires ont été administrés auprès de 270 Mbororo dans les trois arrondissements de la ville de Bafoussam et 230 dans la ville de Dschang.

Les données recueillies sur le terrain ont été traitées et analysées à l'aide des logiciels Statistical Package of Social Science19 (SPSS 19) et Excel 2007 pour la saisie des données et l'élaboration des figures et tableaux ; MapInfo Professional 6.5 et Clobal Mapper 13 pour l'élaboration des cartes.

\section{Résultats}

\section{Métiers exercés par les Mbororo en ville}

Les Mbororo s'intègrent à l'économie urbaine en exerçant des métiers de différentes sortes. Ils sont des commerçants, des cafetiers, des chauffeurs, des gardiens, des vétérinaires, des informaticiens, des militaires entre autres. Autant d'activités qui démontrent leur dynamisme à s'intégrer économiquement (Tableau 1).

Tableau 1 : Métiers exercés par les Mbororo dans les villes de Bafoussam et Dschang

\begin{tabular}{|c|c|c|c|}
\hline Métiers & & Bafoussam & Dschang \\
\hline Commerçant & Pourcentage (\%) & $\mathbf{3 3 , 3 3}$ & 38,82 \\
\hline Bouvier & Pourcentage $(\%)$ & 0,97 & 1,18 \\
\hline Taximen et moto-taximen & Pourcentage $(\%)$ & 16,91 & 8,24 \\
\hline Gardien & Pourcentage $(\%)$ & 1,45 & 10 \\
\hline Surveillant & Pourcentage $(\%)$ & & 0,59 \\
\hline Agriculteur & Pourcentage $(\%)$ & & 3,53 \\
\hline Pâtissier & Pourcentage $(\%)$ & 0,97 & 0,59 \\
\hline Cuisinier & Pourcentage (\%) & & 0,59 \\
\hline Couturier & Pourcentage (\%) & 8,21 & 2,93 \\
\hline Maçon & Pourcentage $(\%)$ & & 0,59 \\
\hline Chargeur & Pourcentage $(\%)$ & 3,86 & 1,18 \\
\hline Fermier & Pourcentage (\%) & & 0,59 \\
\hline Boucher & Pourcentage $(\%)$ & & 10,59 \\
\hline Cafetier & Pourcentage $(\%)$ & 7,73 & 2,94 \\
\hline Musicien & Pourcentage (\%) & 0,48 & \\
\hline Mécanicien & Pourcentage $(\%)$ & 2,9 & \\
\hline Informaticien & Pourcentage $(\%)$ & 0,97 & \\
\hline Vétérinaire & Pourcentage $(\%)$ & 0,48 & \\
\hline Enseignant coranique & Pourcentage $(\%)$ & 0,48 & \\
\hline Coiffeuse & Pourcentage $(\%)$ & 0,48 & \\
\hline Tradi-praticien & Pourcentage (\%) & 1,93 & \\
\hline Infirmier (Ière) & Pourcentage $(\%)$ & 0,97 & \\
\hline Militaire & Pourcentage $(\%)$ & 0,48 & \\
\hline Pompiste & Pourcentage (\%) & 0,48 & \\
\hline Aucune activité & Pourcentage (\%) & 16,91 & $\mathbf{1 7 , 6 5}$ \\
\hline
\end{tabular}

Il ressort du tableau 1 que le commerce est le métier le plus sollicité par les migrants Mbororo (33,33\%) à Bafoussam et $(38,82 \%)$ à Dschang. Ils 
sont des vendeurs de remèdes, de vêtements, de beignes, de chevaux, de produits alimentaires, de viande de vaches braisée. Le tableau met également en évidence la variabilité des métiers d'une ville à l'autre. En effet, la ville de Bafoussam offre davantage de possibilités d'emploi par rapport à la ville de Dschang qui est une ville universitaire et agricole. En outre, les Mbororo s'intègrent tant dans le secteur formel qu'informel. Cependant, on remarque un engagement plus prononcé dans le secteur informel à Dschang, contrairement à Bafoussam où on recense une variété d'emplois effectués par ces derniers. Ils s'adonnent à des activités auxquelles on ne les connaissait pas jusqu'ici: infirmier, mécanicien, informaticien, vétérinaire, pompiste, militaire, boulanger, pâtissier, maçon. Cependant, une partie demeure encore au chômage $(16,91 \%)$ à Bafoussam et $(17,65 \%)$ à Dschang.

\section{Processus de sédentarisation}

Les stratégies mises sur pied par les Mbororo afin de mieux intégrer les activités urbaines sont: l'entraide familiale, l'auto-emploi et les candidatures spontanées, tels qu'énumérés ci-après :

Tableau 2 : Stratégies d'intégration aux activités urbaines à Bafoussam et à Dschang

\begin{tabular}{|cccc|}
\hline $\begin{array}{c}\text { Stratégies d'intégration } \\
\text { économique }\end{array}$ & Bafoussam & Dschang \\
\hline \multirow{2}{*}{ Aide familiale/amicale } & Effectif & 65 & 47 \\
\cline { 2 - 4 } & Pourcentage (\%) & 33 & 33 \\
\hline \multirow{2}{*}{ Auto-emploi } & Effectif & $\mathbf{7 3}$ & $\mathbf{7 8}$ \\
\cline { 2 - 4 } & Pourcentage (\%) & $\mathbf{4 2}$ & $\mathbf{5 6}$ \\
\hline \multirow{2}{*}{ Candidatures spontanées } & Effectif & 35 & 15 \\
\cline { 2 - 4 } & Pourcentage (\%) & 20 & 11 \\
\hline
\end{tabular}

Le tableau 2 présente une prépondérance d'effectif dans l'utilisation de l'auto-emploi comme modalité d'accès à un travail à Bafoussam (42\%) et à Dschang (56\%). En effet, grâce à la vente du bétail en campagne et à l'entraide familiale, il est plus facile pour les Mbororo de s'auto-employer, ce qui explique le choix majoritaire du commerce comme activité. L'entraide familiale qui est de $33 \%$ dans les deux villes concerne les aides et services non formels, provenant soit du réseau familial, soit du voisinage, soit des collègues de travail, soit des amis. Elle s'exprime généralement à travers des transferts financiers et des services divers. En outre, les Mbororo optent aussi pour les dépôts de candidatures auprès de potentiels employeurs.

\section{Obstacles d'intégration à l'économie urbaine}

Les principaux obstacles que rencontrent les Mbororo dans le processus d'intégration à l'économie urbaine sont d'ordre scolaire, financier, liés à l'absence de qualifications professionnelles et le frein de la langue. En effet, si aujourd'hui ce peuple a pris conscience de l'importance et des 
avantages de la scolarisation, il n'en demeure pas moins qu'une partie de sa population reste sous-scolarisée, particulièrement les filles. Ainsi, 24\% de jeunes migrantes Mbororo à Bafoussam et $20 \%$ à Dschang n'étaient pas scolarisées en zone de départ. Une fois installées en ville, $12 \%$ d'entre elles à Bafoussam et $4 \%$ à Dschang demeurent non scolarisées. Les raisons de cette sous-scolarisation évoquées pour la zone de départ par les enquêtés à Bafoussam sont : le manque de moyens (45\%), le jeune âge de la fille (44\%) et les tâches domestiques (11\%). À Dschang, ces derniers évoquent comme raisons le mariage (89\%) et les tâches domestiques (11\%).

En outre, à Bafoussam, les jeunes sont scolarisés du primaire au supérieur. Cependant, plus on avance dans les tranches [30-40ans] et [40$50 \mathrm{ans}$, on se rend compte que la limite du cursus scolaire se situe au primaire, avec certains n'ayant suivi exclusivement que des études arabiques et/ou coraniques (Figure 2).

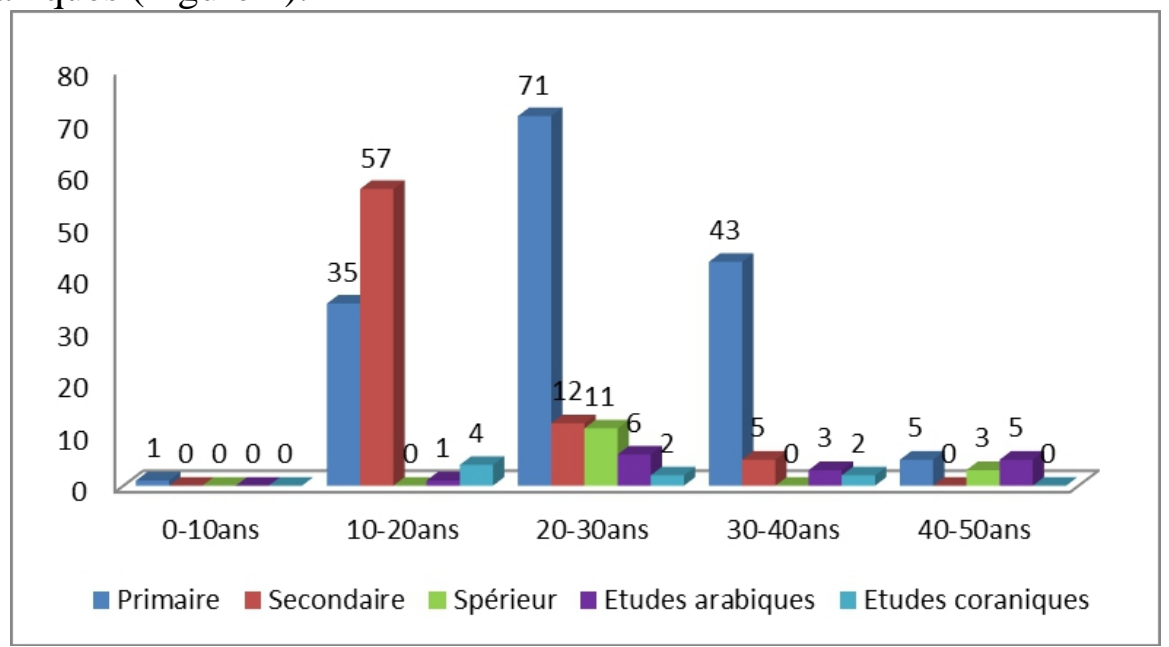

Figure 2 : Niveau d'étude des Mbororo en fonction de l'âge à Bafoussam

On constate par contre que Dschang, ville universitaire, abrite un bon nombre d'étudiants Mbororo. Cependant, tout comme à Bafoussam, les tranches [30-40ans] et [40-50ans] ont arrêté les études au primaire, mais ont reçu des cours exclusivement coraniques. Aucun enquêté se situant dans la tranche [50-60ans] n'a suivi de cursus académique, si ce n'est des études coraniques (Figure 3). 


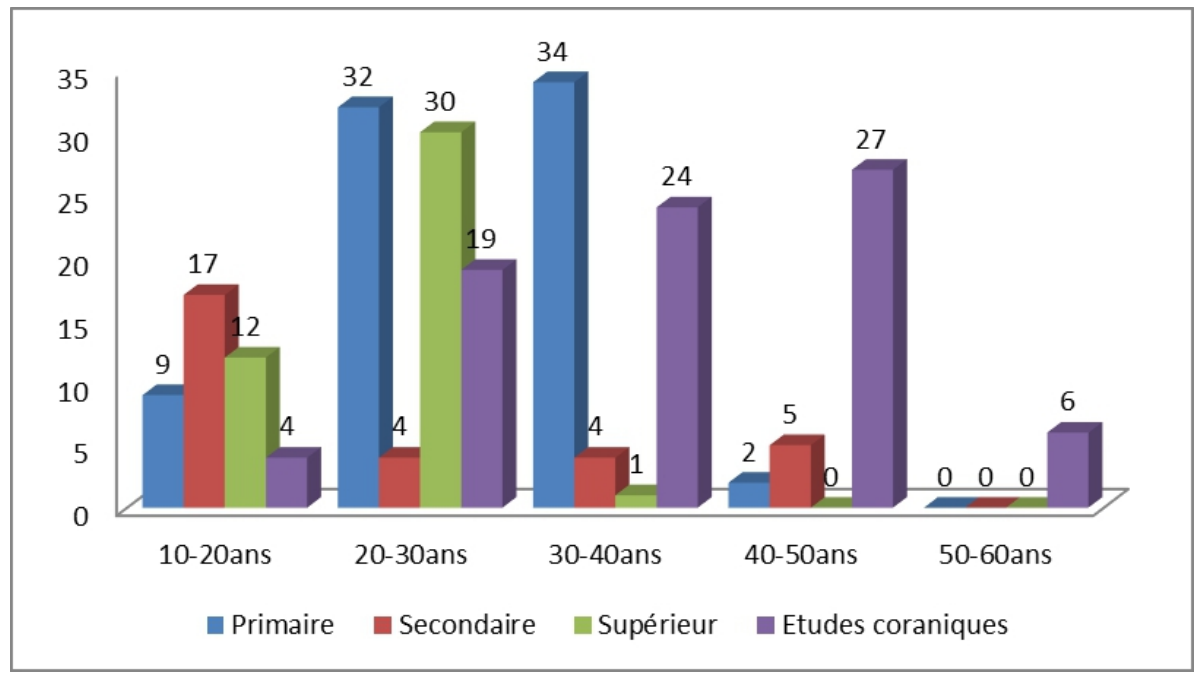

Figure 3 : Niveau d'étude des Mbororo en fonction de l'âge à Dschang

D'autre part, les Mbororo en âge de travailler ont du mal à s'intégrer, à cause de la langue, qui est un réel frein, particulièrement pour les migrants venant des zones rurales de la région du Nord-Ouest, qui s'expriment pour la plupart en anglais, en pidgin et en fulfulde. De plus, outre l'obstacle lié à la sous-scolarisation et au manque de qualification professionnelle, aucun des enquêtés n'est au courant et n'a eu recours même s'il en existe, aux solidarités publiques (aides ou services plus ou moins formels provenant principalement de l'Etat).

\section{Discussion}

Face à la crise du nomadisme pastoral, les Mbororo migrent pour les villes où ils s'intègrent aux activités urbaines. Or ce peuple ne représente pas seulement une société pastorale, mais une société qui au cours du temps a établi une certaine relation entre l'homme et l'animal. Herskovitz (1926), parle d'élevage sentimental. Aujourd'hui, la sédentarisation les pousse à pratiquer des activités sans rapport direct avec l'élevage. Dans la ville de Boromo par exemple, les peuls exercent des activités comme le commerce et l'artisanat. Cependant, ils ne sont pas tous regroupés dans le secteur informel. En effet, quelques rares personnes sont employées dans l'administration comme instituteurs, infirmiers... (Barry 2006). À Bafoussam et à Dschang, on les retrouve tant dans le secteur informel (commerce, gardiennage, couture, cafétéria entre autre), que formel (médecine, enseignement, armée).

Les peuls Mbororo ont accès à ces activités à travers la mise en place d'un certain nombre de stratégies : des processus de sédentarisation. Dans les villes enquêtées, ils s'auto-emploient, mettent en exergue le lien familial et social et se rendent directement auprès de potentiels employeurs. Sandron 
(1998), va dans le même sens en démontrant que l'intérêt du groupe surpasse l'intérêt individuel. Pour cet auteur, dans une tribu, l'existence d'un puissant système d'entraide, social et économique, entre les membres de la tribu est primordiale. «La famille nucléaire n'est en effet pas à même d'assurer son autosubsistance sans ce système de solidarité collective. L'esprit communautaire (apbija) est donc la notion clé pour comprendre le fonctionnement de la tribu »(Sandron, 1998). En effet, le migrant peut se reposer sur les réseaux mis en place par les précédents migrants pour s'intégrer professionnellement. Il existe plusieurs types de réseaux: la parenté, le voisinage, les amis et les compagnons de travail... Tous remplissent des rôles d'intégration sociale (Zouiten, 1995). De fait, les anciens migrants forment une sorte de « niche économique », où ils sont au courant des demandes de services et peuvent s'entraider dans la recherche d'un emploi (Asuncion, 2014).

On note qu'il est possible d'opposer deux types de modes d'insertion : les modes formels, regroupant essentiellement les procédures marchandes (candidatures spontanées et petites annonces) et les modes informels découlant de la mobilisation des liens sociaux (Mareva, 2003). Les Mbororo des villes de Bafoussam et de Dschang mettent en exergue ces deux modes : l'auto-emploi et les candidatures spontanées; l'entraide familiale.

Les enquêtes ont cependant relevé des freins à ce processus d'intégration aux activités urbaines, parmi lesquels la sous-scolarisation chez les plus âgés, qui pour certains ont arrêté les études au primaire, ou n'ont reçu que des enseignements arabiques ou coraniques. Ce qui explique les difficultés à s'exprimer dans un français ou un anglais correct. Ce taux élevé de sousscolarisation chez les Mbororo adultes s'explique par le manque d'intérêt que ces derniers accordaient jadis à la scolarisation moderne. Aujourd'hui, au Tchad par exemple, les obstacles à cette scolarisation sont : le manque de compréhension de la population nomade vivant en petites communautés ethniques isolées, l'irrégularité dans la prise en charge des maîtres communautaires par les parents nomades, la rareté des enseignants issus des communautés nomades, le manque de pâturages qui rend indispensable la mobilité et donc l'absence d'école. Les filles sont particulièrement les plus vulnérables à ces facteurs. Elles font des travaux domestiques non négligeables à partir d'un jeune âge, elles sont parfois en insécurité, avec les grandes distances à parcourir pour se rendre à l'école et enfin, les fiançailles qui interviennent très tôt, parfois à partir de 8 à 10 ans et le mariage précoce découragent également ces dernières à aller à l'école (Swift et al., 2010). Ceci confirme partiellement les résultats obtenus dans ce présent travail, car à ces facteurs, s'ajoute le manque de moyens et le jeune âge de la fille.

Cependant, les Mbororo accordent de plus en plus de l'intérêt à la scolarisation. Barry (2006) va dans ce même sens en démontrant que la plupart des peuls qui ont scolarisé leurs enfants avancent «qu'il faut un minimum 
d'instruction pour se faire une place dans la société ». Un autre frein à cette intégration est l'absence de financement et de suivi. En effet, aucune politique ne semble être mise en place afin d'accompagner ce peuple dans cette transition, raison pour laquelle ils font appel aux solidarités familiales. Or les solidarités privées ou familiales sont plus efficaces lorsque les solidarités publiques existent et fonctionnent bien (Thibault, 2010).

\section{Conclusion}

La présente étude a permis de mettre en exergue le processus d'intégration des migrants Mbororo à l'économie urbaine à Bafoussam et à Dschang dans l'Ouest-Cameroun. Les résultats montrent que les Mbororo sont des commerçants, Taximen et moto-taximen, couturier, enseignants, entre autre. Autant d'activités qui démontrent leur dynamisme à s'intégrer en ville. Afin de faciliter cette intégration, ils ont recours à des stratégies : 1'autoemploi, l'entraide familiale/amicale et les candidatures spontanées. Ces processus de sédentarisation rencontrent toutefois des freins dont la sousscolarisation, la barrière de la langue, l'absence de qualification professionnelle et de financement

Afin de faciliter le processus d'intégration de ce peuple rural, l'on recommande une meilleure visibilité des programmes mis sur pied par l'Etat. Certes ces programmes existent, mais sont totalement méconnus des Mbororo. L'on recommande aussi une accentuation de la promotion de la scolarisation, $\mathrm{du}$ garçon comme de la fille, suivie d'une intensification des formations professionnelles pour toutes les couches sociales. Il serait aussi nécessaire de faciliter et de vulgariser les emprunts et financements pour la mise sur pied des projets de toute nature.

\section{References :}

1. Asuncion, F., (2014). Un double ancrage : liens familiaux et insertion sociale des enfants d'immigrés philippins en France. In : Revue Internationale Enfances, Familles, Générations, 24 p.

2. Barry, H., (2006). Etude des pratiques scolaires des peuls en zone de migration: le cas du département de Bororo. Université d'Ouagadougou, $90 \mathrm{p}$.

3. Boutrais, J., (1996). Population et environnement pastoral en Afrique tropicale. In: Gendreau Francis (ED.), Gubry Patrick (ED.), Véron J., Keyfitz N. (pref.) Populations et environnement dans les pays du Sud, Paris (FRA); Paris : Karthala; CEPED, 1996, (Economie et Développement). ISBN 2-86537-670-2, pp. 177-198.

4. Boutrais, J., (1994). Les Foulbé de l'Adamaoua et l'élevage : de l'idéologie pastorale à la pluriactivité. In Cahiers d'études africaines, Vol. $34 \mathrm{~N}^{\circ} 133-135$, pp. 175 à 196. 
5. Boutrais, J., (1998). Hautes terres d'élevage au Cameroun. In : Annales de Géographie, t.107, n603, 1998, pp. 564-566.

6. ERA-Cameroun, PSEAU et PDM, (2006). Elaboration d'une stratégie concertée d'accès à l'eau et l'assainissement dans la ville de Dschang, rapport final, 108p.

7. Gente, R., (2013). Développement des mines et sédentarisation des nomades en Mongolie Yourtes de banlieue à Oulan-Bator. Parue dans le Monde diplomatique, pp.16 à 17.

8. Herskovitz, M.J., (1926). The Cattle Complex in East Africa. In: American Anthropologist 28 (1): 230-272.

9. Mareva, S., (2003). Stratégies d'insertion et durée d'accès au premier emploi une analyse microéconomique sur le plan téléphonique (198993), 2003/5 Vol.113, pp. 671 à 696.

10. ME de Bruijin, Van, K.O., Obono, O., Oumarou, A., Boureima, D., (2011). Mobilités nouvelles et insécurités dans les sociétés nomades Fulbé (peules) : Étude de plusieurs pays en Afrique Centrale de l'ouest (Niger-Nigeria), African Studies Centre, $57 \mathrm{p}$.

11. Mpakam, H.G., Kamgan, K.B.V., Kouam, K.G.R., Bemmo, N., \& Ekodeck, G.E., (2006). L'accès à l'eau potable et à l'assainissement dans les villes des pays en développement (cas de Bafoussam au Cameroun). Vertigo, Revue en sciences de l'environnement, $\operatorname{Vol} 7 n^{\circ} 2$, Art 12, Septembre 2006, 10p.

12. Piguet, F., (1998). Marginalisation et stratégies de résistance des nomades dans la corne de l'Afrique. In : Open Edition books, pp. 263283.

13. Sandron, F., (1998). L'immobilité forcée : la sédentarisation des nomades dans le Sud Tunisien. Démographe, ORSTON, El Menzah, Tunisie, pp. 63 à 77.

14. Swift, J., Youssouf, A., Mandah, R., Hindou, O., \& Fatimath, A., (2010). Education en milieu pastoral, $39 \mathrm{p}$.

15. Thibault, N., (2010). L'entraide et la réciprocité des échanges familiaux, idées économiques et sociales 4/2010 ( $\left.\mathrm{n}^{\circ} 162\right)$, p 4-5.

16. Zouiten, M., (1995). Migrations, réseaux familiaux et stratégies d'insertion urbaine des migrants ruraux au Maroc, Geography, Université de Montréal, 234p. 